\title{
A PROJECT OF ASTRONOMY TEACHING IN THE SECONDAR SCHOOLS
}

\author{
N.S. Nikolov and T. Stefanova \\ Department of Astronomy, University of Sofia, A. Ivanov Str. 5, 1126 Sofia, Bulgaria
}

At present, astronomical teaching in the Bulgarian secondary schools is incorporated in the subject of physics. This situation dates from about 10 years ago. Before that, astronomy was a separate school subject with 15 to 30 hours in the last (10th) year of school. Now a new reform aroused by the rapid social and economic progress is in operation. During the course of the reform, one not very definitive decision was taken by the Ministry of Education to again separate astronomy, with about 30 hours in the 11th year of a school system which now has 12 years. In connection with this reform, the Ministry asked the Department of Astronomy at Sofia University to work out a project for developing a didactic system for teaching astronomy in the secondary schools. The elements of the system are: alternative variants for the curriculum and the respective textbooks; advisable teaching methods with the appropriate didactic means (diagrams, models, slides, films, etc.); computer programs to demonstrate astronomical objects and phenomena and for pupil computer dialogues, including means for controlling the knowledge level.

In order to find out what was essential:

1) We examined the teaching of astronomy in some other countries.

2) We examined past teaching of astronomy in our country and especially the school textbooks used during the last century and a half (Bulgarian schools started to operate more than a century before liberation from the Turkish yoke).

The studies from these items allowed us to discover a tendency to increase the astrophysical content of school curricula over time. This conclusion led us to pay special attention to the formation of basic astrophysical concepts in school courses in astronomy. For the formation of these concepts, we decided to apply the concept of theoretical generalization from the Soviet pedagogical literature.

3) We carried out a pedagogical experiment for settling the level of astronomical knowledge in the conditions of the present-day school system as well as finding the typical mistakes that pupils make in assimilating astronomical lessons. This establishing experiment was the first stage of a whole pedagogical experiments, respectively.

Because of the similarities in the methods of these three experiments or three stages of our pedagogical experiment, let us now concentrate on some details:

1) The experiment covered about 1200 school students from 12 schools of all kinds all over the country.

2) As a main subject of examination, we chose the stars and, more concretely, 
their essential characteristics - temperature (spectra), luminosity (absolute magnitude), mass, etc.

3) For each characteristic, we gave three stages of problems graded in difficulty. The first stage of problems could be solved with only a simple reproduction of the perceived essential knowledge of the suitable lesson. The second stage - normal problems - one could solve by using the perceived knowledge. The third stage of problems could be solved only by means of creative reflection. That is why we call these creative problems.

4) In order to provide numerical evaluations, we decomposed each problem and its solution into their basic elements. We defined a coefficient $\kappa$ (the relation between the number of true solved elements in a concrete solution and the total number of elements) as a grade for each problem given to the pupils.

The internal experiment held in 1985 (stage 1) showed the following average coefficients for the reproductive and creative problems in the characteristics luminosity, temperature, and mass, respectively:

$\begin{array}{lccc} & \text { Luminosity } & \text { Temperature } & \text { Mass } \\ \text { Reproductive } & 0.73 & 0.63 & 0.36 \\ \text { Creative } & 0.30 & 0.23 & 0.16\end{array}$

These results show that it is necessary to use as much as possible methods that require the continuous active participation of pupils, often placing them in situations that need creative reflection. In addition, the experiments showed that pupils do not perceive sufficiently deeply the physical essence of stellar characteristics and of astronomical notions in general.

With respect to these conclusions, we worked out two variants of the system for the educational experiment. The variants concern mainly the structure of the educational material. We divided each variant into subvariants, differing predominantly in the method of teaching and respective visual means, devices, etc.

In Fig. 1 we show partially the results:

a) The coefficient $\kappa$ for the temperature (spectrum) of stars from the educational experiment held in 1986/1987;

b) The mean $\kappa$ for the three years.

For the rest of the characteristics, the results are similar. The quantity $\eta$ gives the relation of $\kappa$ for the experimental classes to $\kappa$ for the reference classes. We believe that the increase of $\eta$ from the reproductive to the creative level is due at least partially to the system developed by us.

In the forthcoming third stage - the definitive experiment that follows from the three-year educational experiment - positive elements would be used, such as suitably thought-provoking problems given by the teachers to students, selected by 
experimentation in previous years. After that, we would recommend the system to the Bulgarian Ministry of Education.

In conclusion, it is necessary to mention that the system for teaching astronomy at the secondary school level in the People's Republic of Bulgaria developed by our Department would certainly permit corrections, improvements, and ameliorations.

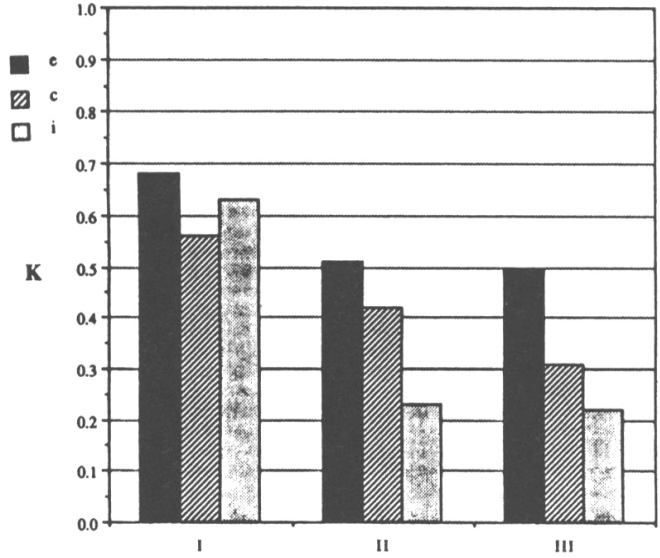

(a)

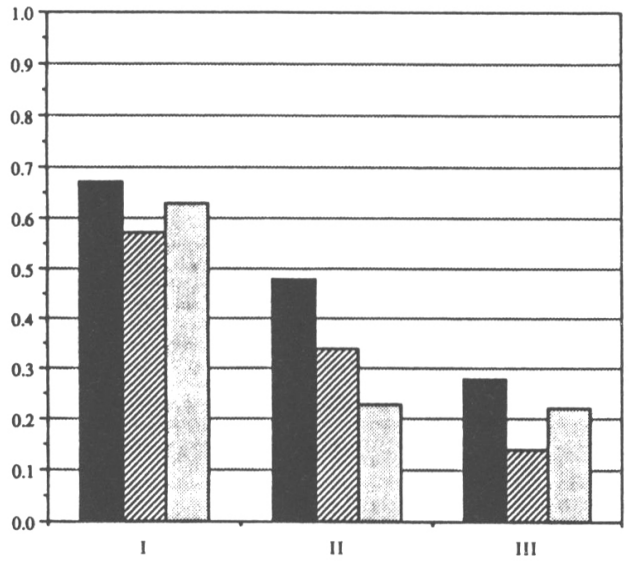

(b)

Fig. 1. Some results of the experiments about the knowledge about the concept star temperature (spectral type, color) (a) from the school year 1986/87; (b) the average from three years of experiment. The number $\kappa$ shows the ratio between the correctly solved elements and the total numbers of elements for solving the problems given to pupils. The symbols denote: $i$ - inference experiment; $e$ - experimental classes; $c$ - control classes for reproductive (I), normal (II), and creative (III) levels of knowledge, respectively. The number $\eta$ shows the ratio between $\kappa$ for the experimental and control classes.

The values of $\eta$ are: (a) $\eta(I)=1.21, \eta(I I)=1.21, \eta(I I I)=1.65$ (b) $\eta(I)=$ $1.20, \eta(I I)=1.53, \eta(I I I)=2.00$. 